\title{
The Electrical Analogue Computer of Microtubule's Protofilament
}

\author{
M. C. Ekosso ${ }^{1},{ }^{1}$ A. J. Fotue, ${ }^{1}$ S. C. Kenfack, ${ }^{1}$ H. Fotsin, ${ }^{2}$ and L. C. Fai ${ }^{1}$ \\ ${ }^{1}$ Mesoscopic and Multilayers Structures Laboratory, Department of Physics, Faculty of Science, University of Dschang, \\ P.O. Box 479, Dschang, Cameroon \\ ${ }^{2}$ Laboratory of Electronics and Signal Processing, Department of Physics, Faculty of Science, University of Dschang, P.O. Box 67, \\ Dschang, Cameroon
}

Correspondence should be addressed to M. C. Ekosso; christekosso@gmail.com

Received 2 July 2020; Revised 6 September 2020; Accepted 13 September 2020; Published 27 September 2020

Academic Editor: Giancarlo Consolo

Copyright ( $) 2020$ M. C. Ekosso et al. This is an open access article distributed under the Creative Commons Attribution License, which permits unrestricted use, distribution, and reproduction in any medium, provided the original work is properly cited.

\begin{abstract}
Microtubules as essential biopolymers implicated into electrical intracellular transport open a lot of questions about their intrinsic character of dynamic instability. Both experimental and theoretical investigations are used to understand their behavior in order to mimic and build powerful and smart biomaterials. So, in this paper, by analytical and computational approaches, we proposed an electrical analogue computer of microtubule's protofilament drawing from the partial differential equation which describes microtubule's motion. Using the computing elements, namely, operational amplifiers, capacitors, and resistors, we designed analytically the bioelectronic circuit of the microtubule's protofilament. To validate our model, Runge-Kutta code was used to solve the partial differential equation of MT's motion on software Matlab, and then, the results obtained are used as a controller to fit and validate numerical results obtained by running the bioelectronic circuit on software PSpice. It is shown that the analogue circuit displayed spontaneous electrical activity consistent with self-sustained electrical oscillations. We found out that two behaviors were exhibited by the voltage generated from the electrical analogue computer of MT's protofilament; amplification and damping behaviors are modulated by the values of the resistor of the summing operational amplifier. From our study, it is shown that low values of the resistor promote damping behavior while high values of the resistor promote an amplification behavior. So microtubule's protofilament exhibits different spontaneous regimes leading to different oscillatory modes. This study put forward the possibility to build microtubule's protofilament as a biotransistor.
\end{abstract}

\section{Introduction}

At the nanoscale, biological systems displayed electrical activity that cares biological communication via electrical signals [1]. Microtubules (MTs), made up of electrically polar tubulin heterodimer subunits $\alpha$-tubulin (negatively charged) and $\beta$-tubulin (positively charged) monomers forming an electric dipole are essential cellular biopolymers, playing an important role in the intracellular signaling and information processing [2], recognized to participate in eukaryote vesicle trafficking and cell division, implicated in cognitive processing by contributing to neuronal developmental structures such as axons and dendrites [3-5]. MTs contribute to the processing of electrical activity of excitable cells and signals within neuron via ion channel regulation [6-8]. They are the source of electromagnetic fields in the form of electric pulses [9]. In that way, they are good conductors of electrical signals at nanolevel and are assimilated to cellular automata $[10,11]$. Besides, microtubules perform mechanical dynamics revealing vibration characteristics and nanoscale effects on their structure [12-16]. Recently, some research studies summarize the possibility of MTs to application in novel bioinspired nanoelectronic components [17]. In fact, because of their self-organization intrinsic character, through dynamic instability phenomenon modulated by temperature physical control, they facilitate the fabrication of nanowires, nodes, and networks in the future for bionanotechnology applications [18]. The field of nanotechnology applications of biomolecular motors is a young and evolving area of research. While the field has advanced significantly in recent decades, it is fraught with several engineering challenges. Both experimental and theoretical 
approaches are used to conduct nanotechnology research on MT. In 2020, Kalra et al. quantified at physiological tubulin concentration the capacitance and resistance of the microtubule network. They reported that through counterionic condensation, microtubules act as charge storage devices across a broad frequency spectrum [19]. Tuszynski et al. , by a theoretical basis and experimental support, showed that MTs behave consistently with the definition of a memristor. They provided an estimation of MT memristance and discussed the significance of biology, especially neuroscience, and the potential for nanotechnology applications [20]. In 2006, Priel et al. investigated MT electrodynamics properties. They reported that MTs behave as biomolecular transistors capable of amplifying electrical information [8]. Recently, Gutierrez et al. reported that isolated brain MTs are electrical oscillators that behave as "ionic-based" transistors and amplify electrical signals which may have important implications in neuronal computational capabilities [21]. All the abovementioned studies have been done experimentally. In the theoretical viewpoint, MT's bioelectronic circuits were first established by Sekulié and Satarić, who was inspired by electrical nonlinear transmission line: RLC circuit [2]. Moreover, Ilić et al. have modelled microtubules as nanowires capable of enhancing ionic transport using a similar approach than Sekulié [22]. In fact, in the theoretical viewpoint, researchers were inspired by a general RLC-cell circuit by considering the values of elements usually estimated experimentally. This consideration is powerful but not sufficient to generate a realistic and unique circuit characterizing the natural MTs. Thus, in this work, we propose an electrical analogue computer of microtubule by drawing from the partial differential equation describing the dynamics of the system. Composed by electrical computing elements such as resistors, capacitors, and operational amplifiers, an electrical analogue computer is an active network capable of simulating any set of linear and nonlinear partial differential equations. The voltage waveform generated from the electrical analogue computer is usually encoded into the time evolution, providing powerful computational real-time operation and complete parallelism. What we will do is to compare numerical results obtained from the partial differential equation using Runge-Kutta code to numerical results obtained from the analogue computer simulated on PSpice. The paper is organized as follows: Section 2 gives an overview about computing elements; in Section 3, the analogue computer of MT's protofilament is presented, Section 4 investigates numerical results on Matlab and PSpice, and Section 5 concludes the study.

\section{The Computing Elements}

In this section, we summarize the relation between output and input voltages of each computing analogue element that can be useful to design the analogue computer. The relations are derived using the common node laws at the entrance of each operational amplifier (Figure 1).

The principal component in an analogue computer is the operational amplifier. When two impedances $Z_{i}$ and $Z_{f}$ are connected to such an amplifier as shown in Figure 1(a), it can be shown that the input and output voltages to the circuit are related by summing the currents:

$$
i_{1}+i_{2}=0 \Longrightarrow\left(\frac{v_{i}}{Z_{i}}\right)+\left(\frac{v_{o}}{Z_{f}}\right)=0
$$

The output voltage is given by

$$
v_{o}=-\left(\frac{Z_{f}}{Z_{i}}\right) v_{i}
$$

Thus, the quantity $\left(Z_{f} / Z_{i}\right)$ acts as an operator on the input voltage $v_{i}$ to produce the output $v_{0}$. When $Z_{f}$ and $Z_{i}$ are chosen to be two resistors, the operator simply becomes a multiplying constant and equation (1) takes the following form:

$$
v_{o}=-\left(\frac{R_{f}}{R_{i}}\right) v_{i}
$$

If $R_{f}=R_{i}$, the output and the input are inversely related as follows:

$$
v_{o}=-v_{i}
$$

This type of circuit given in equation (4) is known as the inverting operational amplifier because of the phase inverting property (negative sign) associated with it. Most inverting amplifiers, which are part of the standard computing setup, usually have multiplication constants of 1,4 , and 10. In general, the amplifier may have more than one input port as shown in Figure 1(b). The result is a summing amplifier, whose output $v_{o}$ is given by

$$
v_{o}=-R_{f}\left(\frac{v_{1}}{R_{1}}+\frac{v_{2}}{R_{2}}+\frac{v_{3}}{R_{3}}\right) .
$$

When a capacitor $C$ and a resistor of resistance $R_{i}$ are connected as shown in Figure 1(c), the output voltage is evaluated as follows:

Assuming $i_{1}+i_{2}=0 \Longrightarrow i_{1}=-i_{2}$

$$
i_{2}=C \frac{\mathrm{d}\left(v_{o}-v_{-}\right)}{\mathrm{d} t}=C \frac{\mathrm{d} v_{o}}{\mathrm{~d} t} .
$$

Knowing that $v_{-} \longrightarrow 0$

$$
i_{1}=\left(\frac{v_{i}-v_{-}}{R_{i}}\right)=\left(\frac{v_{i}}{R_{i}}\right) .
$$

Using equations (6) and (7), the corresponding node law relation can be written as follows:

$$
i_{1}+i_{2}=\left(\frac{v_{i}}{R_{i}}\right)+C\left(\frac{\mathrm{d} v_{o}}{\mathrm{~d} t}\right)=0 .
$$

From equation (8), the output voltage is given by the following expression:

$$
v_{o}=-\left(\frac{1}{R_{i} C}\right) \int v_{i} \mathrm{~d} t .
$$

In the case of many input voltages, the output is evaluated by summing all the input currents (Figure 1(d)): $i_{1}+$ 


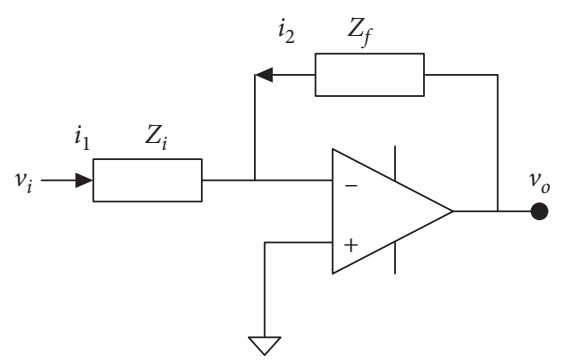

(a)

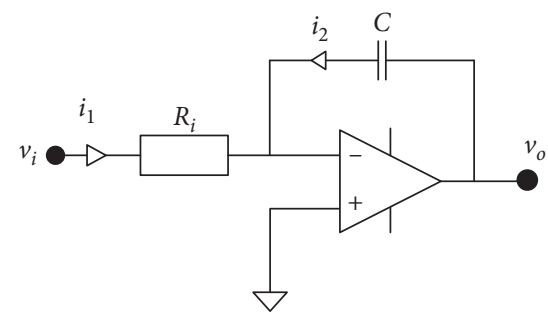

(c)

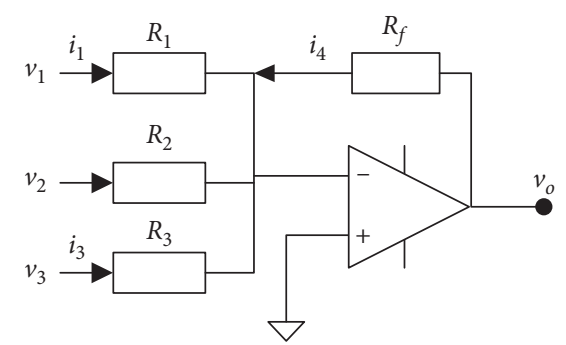

(b)

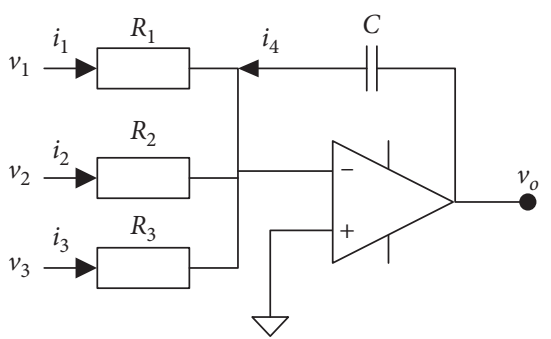

(d)

Figure 1: Computing elements. (a) High gain dc amplifier with feedback and input impedances, (b) summing amplifier, (c) integrating amplifier, and (d) summing integrating amplifier.

$i_{2}+i_{3}+i_{4}=0$, with $i_{1}=C\left(\mathrm{~d} v_{o} / \mathrm{d} t\right), i_{3}=\left(v_{2} / R_{2}\right), i_{2}=\left(v_{1} / R_{1}\right)$, $i_{4}=\left(v_{3} / R_{3}\right)$

So $C\left(\mathrm{~d} v_{o} / \mathrm{d} t\right)+\left(v_{1} / R_{1}\right)+\left(v_{2} / R_{2}\right)+\left(v_{3} / R_{3}\right)=0$, the output voltage in this case is expressed as follows:

$$
v_{o}=-\left(\frac{1}{C}\right) \int\left(\left(\frac{v_{1}}{R_{1}}\right)+\left(\frac{v_{2}}{R_{2}}\right)+\left(\frac{v_{3}}{R_{3}}\right)\right) \mathrm{d} t \text {. }
$$

\section{Analogue Computer of MT's Protofilament}

In this section, the differential equation describing the motion of a protofilament is presented. The time encode equation is then expressed as 2 parametric equations. By using these parametric equations and the suitable computing components summarized above, the electrical analogue circuit is designed. From the designed circuit, the electrical equation of motion is performed and compared to the original differential equation in order to derive the network of resistors necessary to run the circuit on PSpice.

3.1. Differential Equation of Motion. The model assumes only one degree of freedom per dimer, which is common for, more or less, each model describing microtubule dynamics. According to the model, the dimers perform angular oscillations, but the coordinate $u$ is the projection of the top of the dimer on the direction of protofilament and the model can be regarded as the longitudinal one $[23,24]$. The general equation describing MT's protofilament motion using Hamilton equations is expressed as follows:

$$
\alpha\left(\frac{\mathrm{d}^{2} \psi}{\mathrm{d} \xi^{2}}\right)-\rho\left(\frac{\mathrm{d} \psi}{\mathrm{d} \xi}\right)-\beta \psi+\delta \psi^{3}-\sigma=0 .
$$

By assuming the dimensionless parameters: $\alpha=\left(\left(m \omega^{2}-k l^{2} \kappa^{2}\right) / A\right), \quad \rho=(\gamma \omega / A), \sigma=(q E / A \sqrt{(A / B)})$, and $\beta \approx \delta \longrightarrow 1$ in which $m$ is a mass of the dimer, $k$ is an intradimer stiffness parameter, $q>0$ represents the excess charge within the dipole, $E>0$ is internal electric field and the integer, $A$ is a constant depending on temperature and $B$ is an arbitrary constant, $\gamma$ is a viscosity coefficient of the cytosol, and $l$ is a length of a tubulin dimer. Since $\zeta=\kappa X-\omega_{0} t, \alpha$ measures the competition between the kinetic energy of the tubulin dimer and the potential energy of pertaining chemical bonds $[23,24], \rho$ is a constant related to viscosity coefficient of the cytosol. Parameters $\beta$ and $\delta$ are arbitrary and related to the nonlinearity of the system. $\sigma$ characterizes the electrical force, and this parameter was evaluated by Zdravkovic et al. [23, 24]; equation (11) depends on both space and time. But in this study, we shall consider only the temporal variation of the wave function generated during the dynamic instability phenomenon of MT. The spatial term in $\xi$ will be considered invariant. So, the time dependence of the equation of motion is written as follows:

$$
\alpha \ddot{x}-\rho \dot{x}-\beta x+\delta x^{3}-\sigma=0 .
$$

$\tau=\omega_{0} t$ and $\dot{x}=(\mathrm{d} x / \mathrm{d} \tau)$, and $x$ represents the part of the wave function that depends only on time. It is assumed that equations (11) and (12) represent a first approximation of the system with a single degree of freedom related to longitudinal displacement.

Let us proceed by parametric equation by supposing $\dot{x}=y$, equation (12) can be written as a set of 2 equations as follows:

$$
\left\{\begin{array}{l}
\dot{x}=y, \\
\dot{y}=\left(\frac{\rho}{\alpha}\right) y+\left(\frac{\beta}{\alpha}\right) x-\left(\frac{\delta}{\alpha}\right) x^{3}+\left(\frac{\sigma}{\alpha}\right) .
\end{array}\right.
$$


So,

$$
\left\{\begin{array}{l}
x=\int y \mathrm{~d} \tau \\
y=\int\left(\left(\frac{\rho}{\alpha}\right) y+\left(\frac{\beta}{\alpha}\right) x-\left(\frac{\delta}{\alpha}\right) x^{3}+\left(\frac{\sigma}{\alpha}\right)\right) \mathrm{d} \tau .
\end{array}\right.
$$

Using equation (13) and considering the computing elements previously study, we can generate the electrical analog computer that corresponds to MT's protofilament equation of motion over time as shown in Figure 2.

In fact, the procedure used to design the circuit is as follows.

Step 1. The circuit will be built using 2 input voltages corresponding to $\dot{x}$ and $\dot{y}$. We have to derive the exact corresponding expression of each input voltage using capacitors and resistors. As shown in equation (14), it is suitable to build $x$ and $y$ using integrating amplifier in Figure 1(c) corresponding to equation (9). Let us use equation (9) to evaluate the input voltage as follows: $v_{o}=-\left(1 / R_{i} C\right) \int v_{i} \mathrm{~d} t \longrightarrow R_{i} C v_{o}=-\int v_{i} \mathrm{~d} t$, meaning that $R_{i} C \dot{v}_{0}=-v_{i}$. We have to keep in mind that if we integrate the input $\dot{x}$ by using equation (9), we shall get $-x$ as an output. Thus, the expression $R_{i} C \dot{v}_{0}=-v_{i}$ will be rewritten as follows: $-v_{i_{1}}=R_{1} C_{1}(-\dot{x})$ for the first parametric equation and $-v_{i_{2}}=R_{2} C_{2}(-\dot{y})$ for the second parametric equation in equation (13) as shown in Figure 2, respectively.

Step 2. By observing the second parametric equation in equation (13), we should generate $y, x, x^{3}$ and a constant input $V_{C C}$ corresponding to a last term of the expression. The first term $y$ is built using inverting amplifier of Figure 1(a) with $Z_{i}=Z_{f}=R$ to inverse the input $-y$ to the output $y$ as expressed in equation (4). The same procedure is done to get $x$ from $-x$. The input $x$ is then multiplied 3 times to get $x^{3}$ as shown in Figure 2 .

Step 3. In this stage, we have to connect all the components together with respect to equation (13). From the first parametric equation, the input $\dot{x}$ will be connected to the output $y$ of the second parametric equation. From the second parametric equation of equation (13), we have to sum $-y,-x, x^{3}$, and $-V_{\mathrm{CC}}$ by using summing amplifier of Figure 1(b) where the corresponding expression of the output is given by equation (5). The input $-V_{\mathrm{CC}}$ is added such that the summing amplifier is constituted by 4 input voltages corresponding to each term. All terms in the second parametric equation of equation (13) are signed negative except $x^{3}$ which is signed positive, as the sign in the formula of equation (5) can suitably be corresponded to the expression of equation (13). $R_{f}$ in equation (5) corresponds to $R^{\prime}$ in Figure 2. The output of the summing amplifier is then connected to the input voltage $R_{2} C_{2} \dot{y}$ to balance the right side to the left side of the second parametric equation of equation (13).
3.2. Derivation of Electrical Equation of Motion. The objective now is to evaluate the corresponding values of each computing component according to MT's parameters (Figure 2). In fact, the electrical equation of vibration of the corresponding circuit of a protofilament is computed by using the inverse procedure.

From Figure 2, we get directly

$$
\left\{\begin{array}{l}
R_{1} C_{1} \dot{x}^{\prime}=y, \\
R_{2} C_{2} \dot{y}^{\prime}=-R^{\prime}\left(\left(\frac{-y}{R_{3}}\right)-\left(\frac{x}{R_{4}}\right)+\left(\frac{x^{3}}{R_{5}}\right)-\left(\frac{V_{\mathrm{cc}}}{R_{6}}\right)\right),
\end{array}\right.
$$

where $\dot{x}^{\prime}=\mathrm{d} x / \mathrm{d} t$, and (15) can be rewritten as follows:

$$
\left\{\begin{array}{l}
\dot{x}^{\prime}=\frac{1}{R_{1} C_{1}} y, \\
\dot{y}^{\prime}=\frac{R^{\prime}}{R_{3} R_{2} C_{2}} y+\frac{R^{\prime}}{R_{4} R_{2} C_{2}} x-\frac{R^{\prime}}{R_{5} R_{2} C_{2}} x^{3}+\frac{R^{\prime}}{R_{6} R_{2} C_{2}} V_{\mathrm{cc}} .
\end{array}\right.
$$

As stated earlier, we make the change of time variable as $\tau=\omega_{0} t \Longrightarrow \mathrm{d} \tau=\omega_{0} \mathrm{~d} t$. Equation (16) is then recast to

$$
\left\{\begin{array}{l}
\omega_{01}\left(\frac{\mathrm{d} x}{\mathrm{~d} \tau}\right)=\omega_{01} \dot{x}=\frac{1}{R_{1} C_{1}} y, \\
\omega_{02}\left(\frac{\mathrm{d} y}{\mathrm{~d} \tau}\right)=\omega_{02} \dot{y}=\left(\frac{R^{\prime}}{R_{3} R_{2} C_{2}}\right) y+\left(\frac{R^{\prime}}{R_{4} R_{2} C_{2}}\right) x \\
-\left(\frac{R^{\prime}}{R_{5} R_{2} C_{2}}\right) x^{3}+\left(\frac{R^{\prime}}{R_{6} R_{2} C_{2}}\right) V_{\mathrm{cc}} .
\end{array}\right.
$$

The final expression can be written as follows:

$$
\left\{\begin{aligned}
\dot{x} & =\frac{1}{R_{1} C_{1} \omega_{01}} y, \\
\dot{y} & =\left(\frac{R^{\prime}}{R_{3} R_{2} C_{2} \omega_{02}}\right) y+\left(\frac{R^{\prime}}{R_{4} R_{2} C_{2} \omega_{02}}\right) x \\
& -\left(\frac{R^{\prime}}{R_{5} R_{2} C_{2} \omega_{02}}\right) x^{3}+\left(\frac{R^{\prime}}{R_{6} R_{2} C_{2} \omega_{02}}\right) V_{\mathrm{cc}} .
\end{aligned}\right.
$$

By identifying equation (13) to (18), we can get the following relations: 


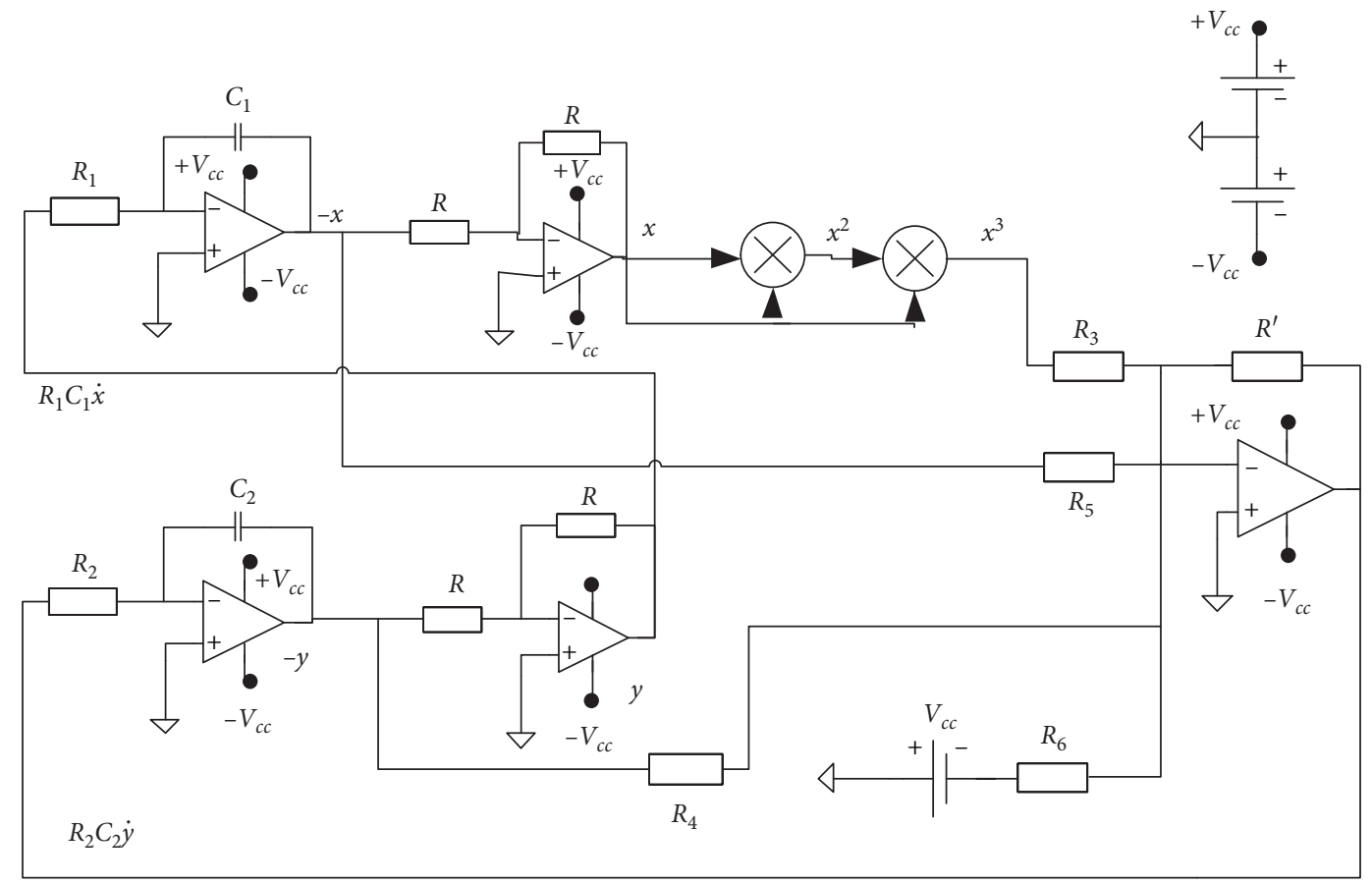

FIGURE 2: Electrical analogue computer of microtubule's protofilament.

$$
\left\{\begin{array}{l}
\left(\frac{1}{R_{1} C_{1} \omega_{01}}\right)=1, \\
\left(\frac{R^{\prime}}{R_{3} R_{2} C_{2} \omega_{02}}\right)=\left(\frac{\rho}{\alpha}\right), \\
\left(\frac{R^{\prime}}{R_{4} R_{2} C_{2} \omega_{02}}\right)=\left(\frac{\beta}{\alpha}\right) \\
\left(\frac{R^{\prime}}{R_{5} R_{2} C_{2} \omega_{02}}\right)=\left(\frac{\delta}{\alpha}\right), \\
R_{3}=\frac{R^{\prime}}{R_{2} C_{2} \omega_{02} *(\rho / \alpha)} \\
\left(\frac{R^{\prime}}{R_{6} R_{2} C_{2} \omega_{02}} V_{c c}\right)=\left(\frac{\sigma}{\alpha}\right) . \\
R_{4}=\frac{R^{\prime}}{R_{2} C_{2} \omega_{02} *(\beta / \alpha)} \\
R_{5}=\frac{R^{\prime}}{R_{2} C_{2} \omega_{02} *(\delta / \alpha)} \\
R_{6}=\frac{R^{\prime} V_{c c}}{R_{2} C_{2} \omega_{02} *(\sigma / \alpha)}
\end{array}\right.
$$

\section{Numerical Results}

In this section, we have solved the equation of motion numerically using Runge-Kutta code and then run the corresponding circuit on PSpice. The purpose of this procedure is to have an idea about the general behavior of the waveforms propagating along protofilament.

By varying the values of $\alpha, \rho, \sigma$ at constant parameters $\beta, \delta$ related to the nonlinearity. The following solutions are obtained.

It is observed that the nature of waves depends on the value of $\alpha$. For low values of $\alpha$, waves generated are subsonic waves as shown in Figures 3 and 4. For high values of $\alpha$, waves generated are supersonic as shown in Figure 5. As previously mentioned, $\alpha$ measures the competition between the kinetic energy and the chemical potential of pertaining bonds. We assume that high values of $\alpha$ mean the highest of kinetic energy in front of chemical potential promotes supersonic behavior while low values of $\alpha$ promote subsonic behavior when the chemical potential of pertaining bonds is higher.

In the case of subsonic behavior (see Figures 3 and, 4), it is shown that by increasing the value of $\alpha$, the wave amplitude increases and oscillations are promoted. Moreover, parameter $\sigma$ that relates to electric force promotes oscillations as well. As parameter $\rho$ related to cytosol viscosity increases, the signal amplitude decreases over time. Parameters $\delta$ and $\beta$ do not affect the signal amplitude; we consider them equal to 1 . So, for subsonic cases, $\alpha$ and $\sigma$ promote oscillations and increase the signal amplitude. The damping parameter $\rho$ collapses oscillations and promotes a linear and constant signal over time. In the case of supersonic behaviour (see Figure 5), it is observed that the increase in $\alpha$ and $\sigma$ do not change the signal amplitude, but it is well observed that their increase promotes oscillations, while the increase of parameter $\rho$ collapses oscillations. In this last case, it is observed that the amplification phenomenon can arise infinitely for high values of $\alpha$ and $\sigma$ and for low values of $\rho$. This situation is an ideal case and difficult to occur in nature in general and particularly in biological systems. Generally, the switch between amplification and damping behaviors of a signal generated cares the transmission process during the dynamics of biological systems. This allows to balance the energy used and procedure in the system. Besides, each transmitted signal is amplified or 


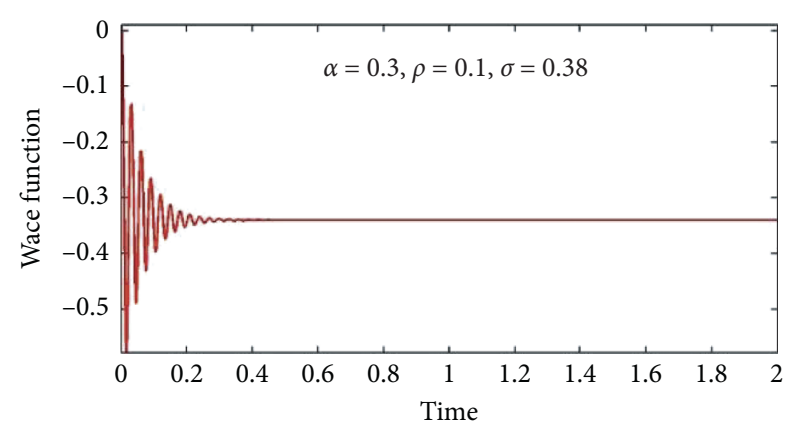

(a)

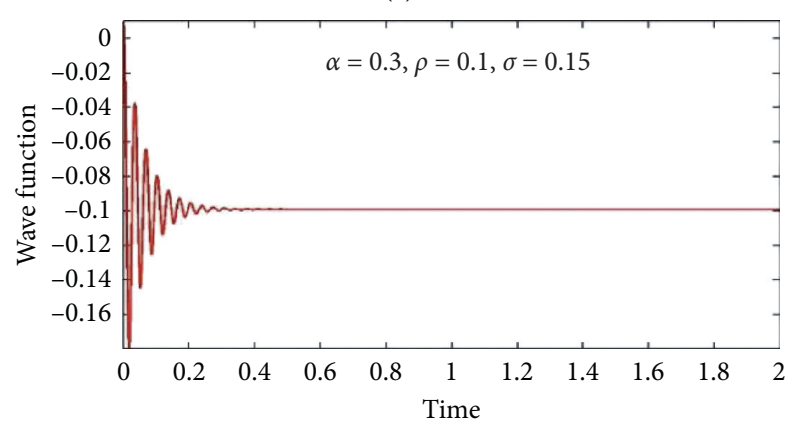

(c)

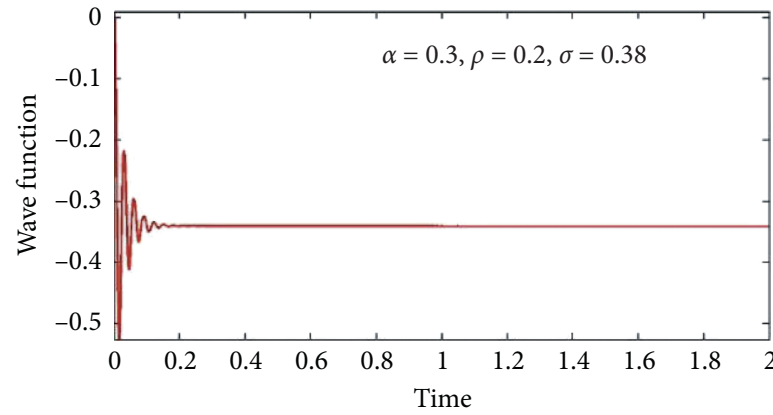

(b)

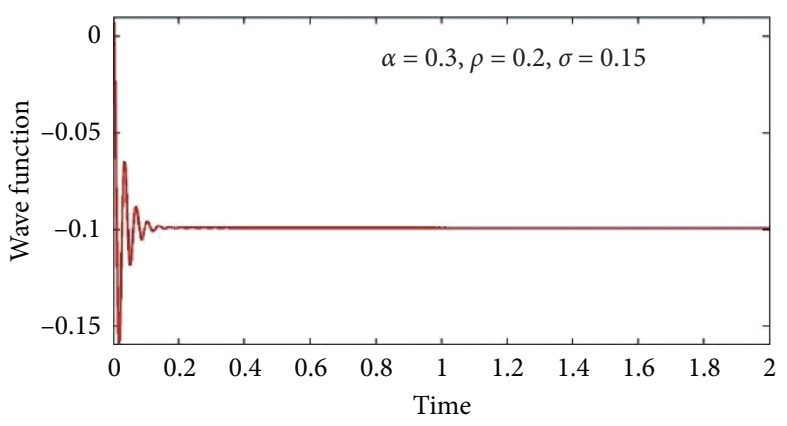

(d)

FIGURE 3: Wave function generated from the partial differential equation at low kinetic energy. (a) $\alpha=0.3, \rho=0.1, \sigma=0.38$; (b) $\alpha=0.3, \rho=0.2, \sigma=0.38$; (c) $\alpha=0.3, \rho=0.1, \sigma=0.15$; (d) $\alpha=0.3, \rho=0.2, \sigma=0.15$.

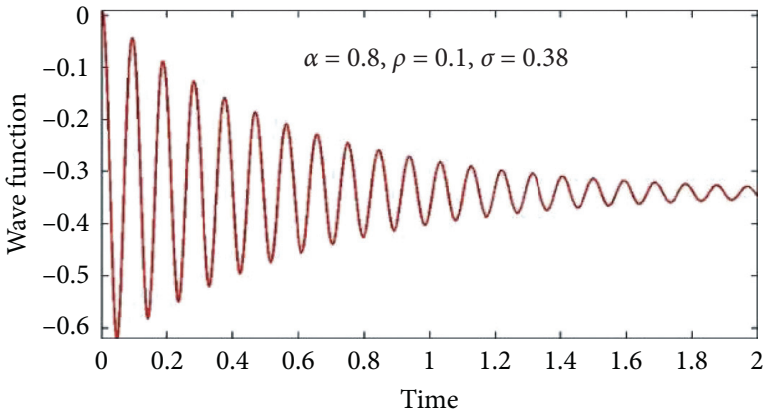

(a)

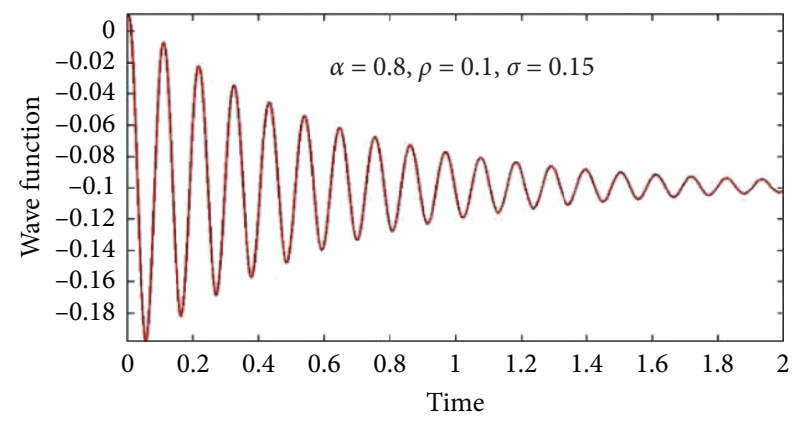

(c)

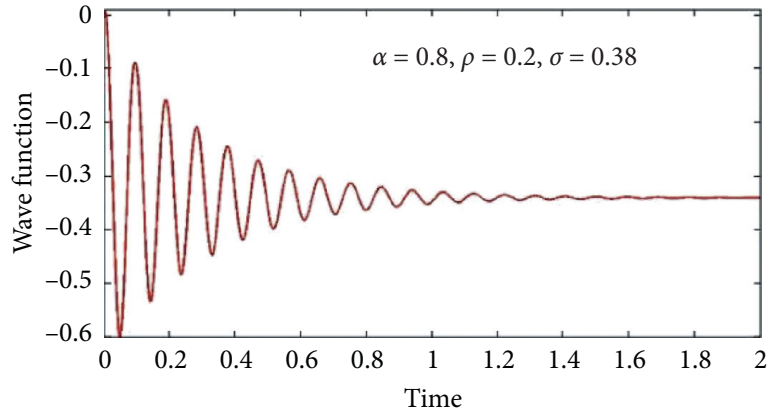

(b)

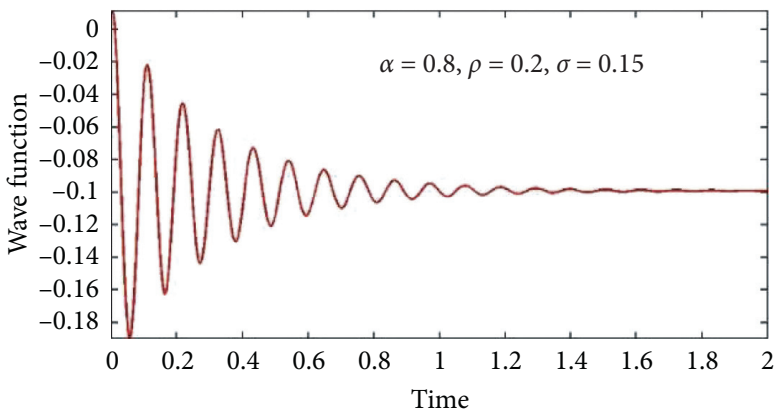

(d)

FiguRE 4: Wave function generated from the partial differential equation at high kinetic energy. (a) $\alpha=0.8, \rho=0.1, \sigma=0.38$; (b) $\alpha=0.8, \rho=0.2, \sigma=0.38$; (c) $\alpha=0.8, \rho=0.1, \sigma=0.15$; (d) $\alpha=0.8, \rho=0.2, \sigma=0.15$.

damped for a given time and then usually becomes constant or totally vanishes or switches to an opposite behavior over time. In the case of the dynamic instability of natural microtubule, we assume that the energy modulating the polymerization/depolymerization cannot care an infinite amplification over time. In fact, the energy produced during 


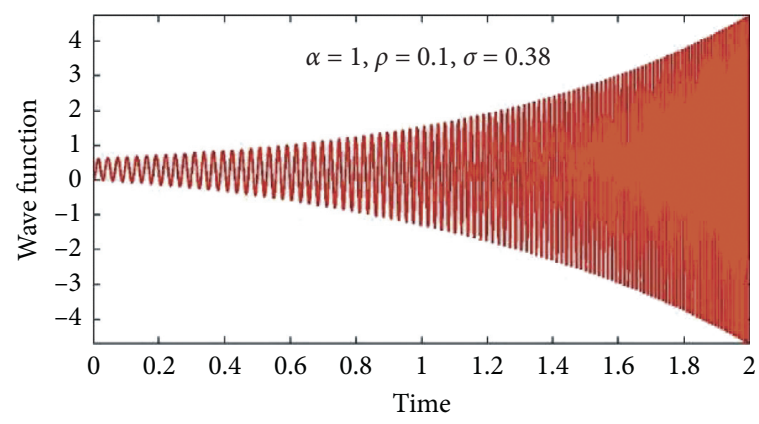

(a)

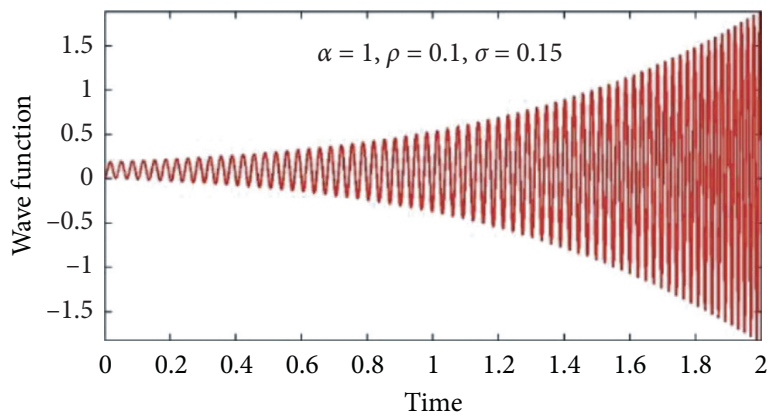

(c)

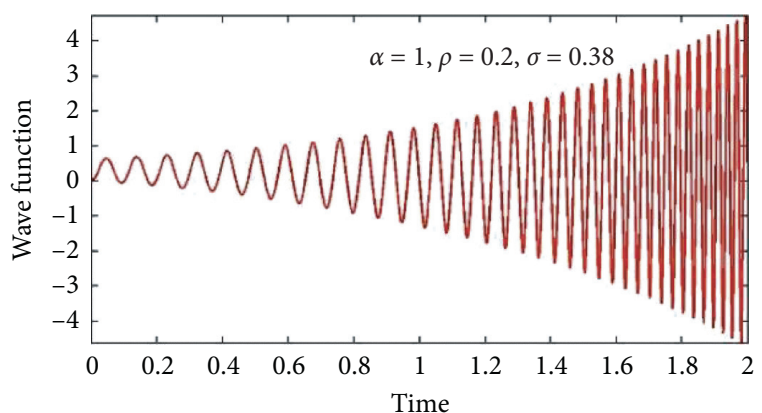

(b)

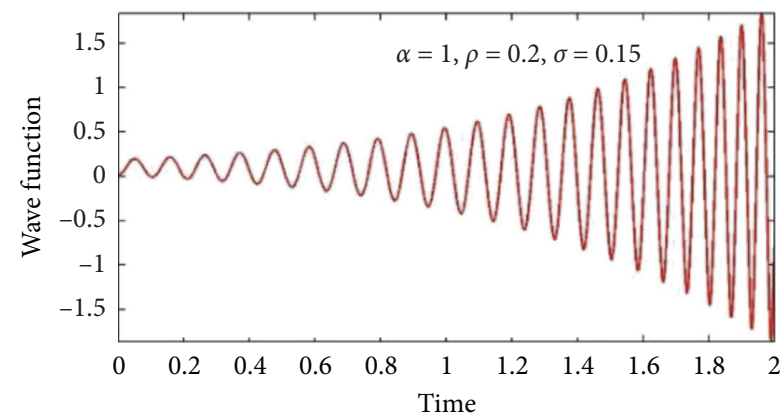

(d)

Figure 5: Wave function generated from the partial differential equation at very high kinetic energy. (a) $\alpha=1, \rho=0.1, \sigma=0.38$; (b) $\alpha=1, \rho=0.2, \sigma=0.38$; (c) $\alpha=1, \rho=0.1, \sigma=0.15$; (d) $\alpha=1, \rho=0.2, \sigma=0.15$.

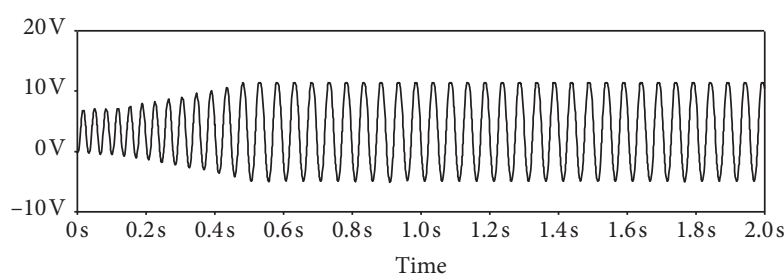

口 V(UC1: OUT)

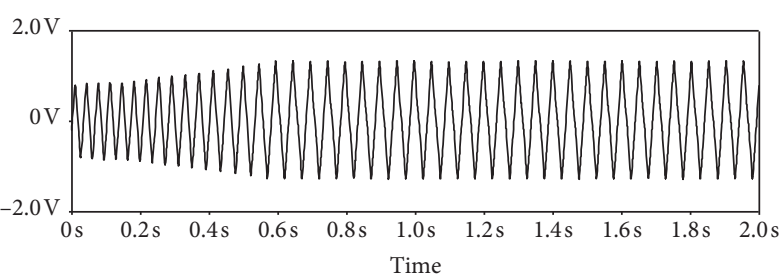

V(UC2: OUT)

(a)

(b)

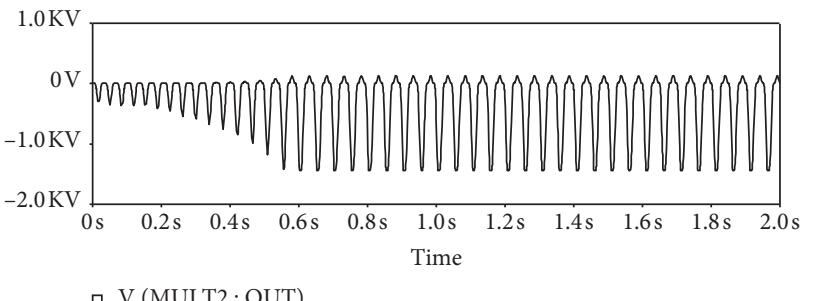

(c)

Figure 6: Output voltages of (a) amplifier with capacitor $C_{1}$, (b) amplifier with capacitor $C_{2}$, and (c) the nonlinear part $x^{3}$ for $R^{\prime}=10 K \Omega$, $C_{1}=100 \mathrm{nF}, C_{2}=10 \mathrm{uF}, R=R_{1}=R_{2}=R_{3}=R_{5}=10 \mathrm{~K} \Omega, V_{\mathrm{cc}}=12 \mathrm{~V}, R_{4}=5 \mathrm{~K} \Omega, R_{6}=2.77 \mathrm{~K} \Omega$.

the polymerization is used during the depolymerization. During this situation, the energy carried by the waveform moving along protofilament varies constantly and the oscillations generated during the vibration are probably affected. So the chemical energy of pertaining bonds can increase or decrease the kinetic energy as the corresponding parameter $\alpha$ remains low. From the above mentioned, we argue that the real system cannot be designed in the situation of very high kinetic energy. Thus to run the electrical analogue on PSpice, we will stay in the situation of Figures 3 and 4 and mimic both the amplification and damping behaviors.

Let us run the electrical analogue computer using the software PSpice by considering appropriate values of each parameter as given in Figures 3 and 4 . The circuit is run for the case where $\alpha=0.3, \rho=0.1, \sigma=0.38$, but similar curves 


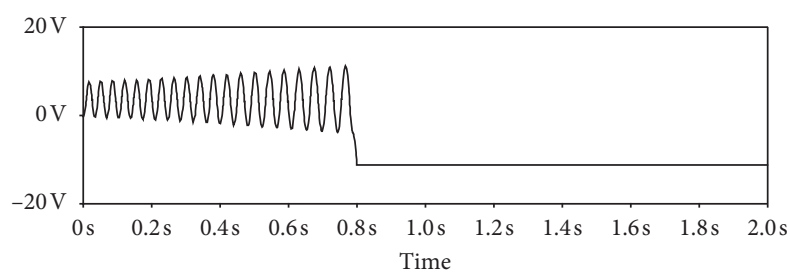

ㅁ $\mathrm{V}(\mathrm{UC} 1:$ OUT $)$

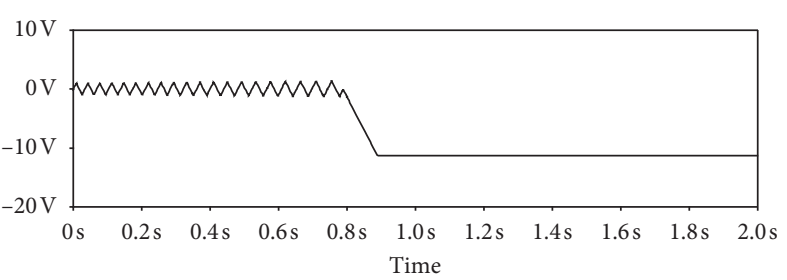

$\mathrm{V}(\mathrm{UC} 2$ : OUT $)$

(a)

(b)

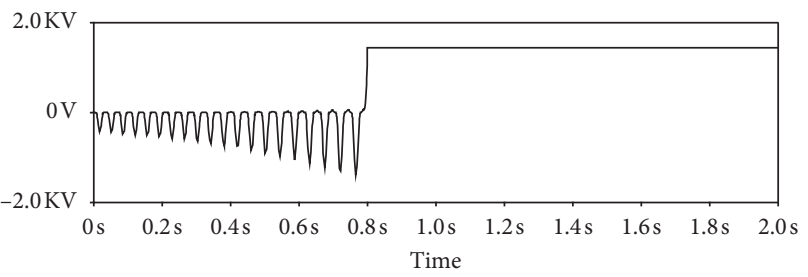

V(MULT2: OUT)

(c)

FiguRE 7: Output voltages of (a) amplifier with capacitor $C_{1}$, (b) amplifier with capacitor $C_{2}$, and (c) the nonlinear part $x^{3}$ for $R^{\prime}>10 K \Omega$, $C_{1}=100 \mathrm{nF}, C_{2}=10 \mathrm{uF}, R=R_{1}=R_{2}=R_{3}=R_{5}=10 \mathrm{~K} \Omega, V_{\mathrm{cc}}=12 \mathrm{~V}, R_{4}=5 \mathrm{~K} \Omega, R_{6}=2.77 \mathrm{~K} \Omega$.

are obtained by using values of $\alpha$ less than 1 and $\sigma \prec \sigma_{\max }=(2 / 3 \sqrt{3})$. These values of parameters are reported in $[23,24]$ and were evaluated within the situation of a favorable configuration that promotes information processing during the dynamic instability.

By varying resistances, we will try to mimic amplifying or damping behaviors from the electrical signal. For the following simulation values $C_{1}=100 \mathrm{nF}, C_{2}=10 \mathrm{uF}$, $R=10 \mathrm{~K} \Omega$ chosen, the signal generated will be convergent. Other values can be used. By fixing these parameters, we use equation (19) to compute other resistances useful for our simulations. The lecturer needs to keep in mind that for each value of $R^{\prime}$, he has to evaluate the corresponding network of resistances.

Numerical results previously obtained from MATLAB (Figures 3-5) are used as a controller to find suitable values of resistors. Suitable values of MT parameters are chosen as the electrical analog computer can mimic the behaviour generated by Runge-Kutta code. Voltage is measured at the output of memory computing elements (amplifiers) and the output of the nonlinear proportion $x^{3}$. The increase or decrease behaviour of the output voltage of the electrical analogue computer is modulated by the values of $R^{\prime}$.

For $R^{\prime}=10 \mathrm{~K} \Omega$ as shown in Figure 6, the output voltages exhibit an increasing behaviour and maintain the same amplitude over time. In Figure 6, spontaneous oscillations showed changes in signal's regime and amplitude, namely, amplification regime where signal's amplitude increases with time from $0 \mathrm{~s}$ to $0.6 \mathrm{~s}$ and constant regime where signal's amplitude remains constant over time (from $0.6 \mathrm{~s}$ to $2 \mathrm{~s}$ ). These results showed the ability of a single microtubule's protofilament to handle amplification of electrical activity and self-controlled the amplification by maintaining the behaviour constant over time. This situation puts forward realistic behaviour of the physical system as previously mentioned. Spontaneous changes are well observed in signal's amplitude, suggesting that intracellular electrical signaling may heavily obey to the assembly and organization of the various cytoskeletal structures over time [21]. For $R^{\prime}>10 K \Omega$ as shown in Figure 7 , the oscillations collapse over time and the output voltages become linear. In fact, by increasing the value of $R^{\prime}$ up to $10 \mathrm{~K} \Omega$, the output voltages also exhibit two regimes: spontaneous amplification of oscillations from $0 \mathrm{~s}$ until $0.8 \mathrm{~s}$ with a clear change in the signal's amplitude and then a constant and linear response from $0.8 \mathrm{~s}$ until $2 \mathrm{~s}$. These changes in regime and amplitude can suggest changes in polarity of the holding potential and conductance as reported by Gutierrez et al. [21] in the case of isolated microtubule.

By decreasing the value $R^{\prime}$ as $R^{\prime} \leq 1 K \Omega$ (Figures 8 and 9), the output voltages exhibit a decreasing behaviour of the oscillation amplitude over time, without becoming linear similar to the behaviour shown in Figures 3 and 4). Results obtained in Figures 8 and 9 are similar to those obtained by Gutierrez et al. [21]. By decreasing the values of $R_{3}$ and $R_{5}$ as shown in Figure 9, the same behaviour as in Figure 8 is obtained. In general, the behavior of the output voltages depends on the values taken by $R^{\prime}$. For $\left.R^{\prime} \in\right] 1 K \Omega-10 K \Omega$ [, the same output voltages as in Figure 6 are generated. According to the results obtained, it is observed that low values of resistance $R^{\prime}$ act as damping parameter but care oscillations over time while high values of resistance $R^{\prime}$ act as amplifier parameter but maintain the amplitude of output constant by increasing time. For these cases, it is also observed that the signal exhibits approximately two regimes as previously (Figure 6). However, an interesting difference became evident, namely, that the richer oscillatory behavior drastically changed to damping one and sustained spontaneous stronger oscillations over time. In fact, it is a well-observed fast spontaneous damping regime, followed by smoothly damping regime over time (Figures 8 


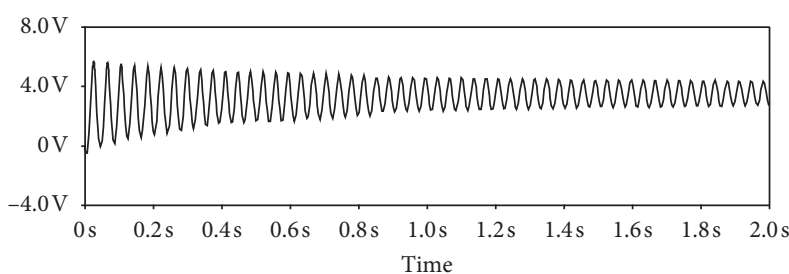

ㅁ $\mathrm{V}(\mathrm{UC} 1$ : OUT $)$

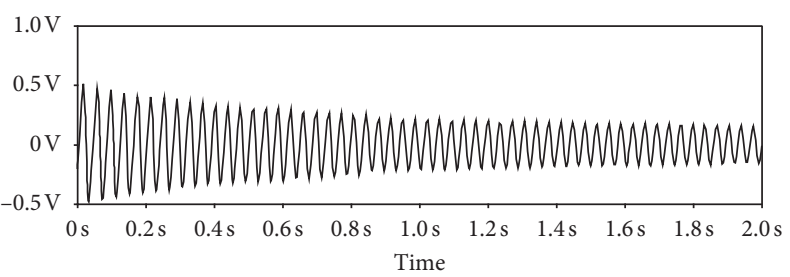

$\mathrm{V}(\mathrm{UC} 2$ : OUT $)$

(a)

(b)

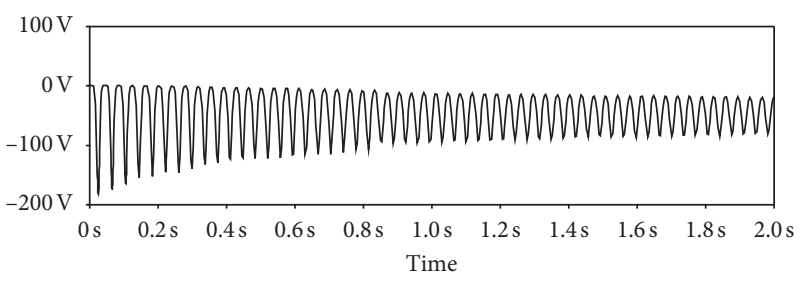

a V(MULT2: OUT)

(c)

FIGURE 8: Output voltages of (a) amplifier with capacitor $C_{1}$, (b) amplifier with capacitor $C_{2}$, and (c) the nonlinear part $x^{3}$ for $R^{\prime} \leq 1 K \Omega$, $C_{1}=100 \mathrm{nF}, C_{2}=10 \mathrm{uF}, R=R_{1}=R_{2}=R_{3}=R_{5}=10 \mathrm{~K} \Omega, V_{\mathrm{cc}}=12 \mathrm{~V}, R_{4}=5 \mathrm{~K} \Omega, R_{6}=2.77 \mathrm{~K} \Omega$.

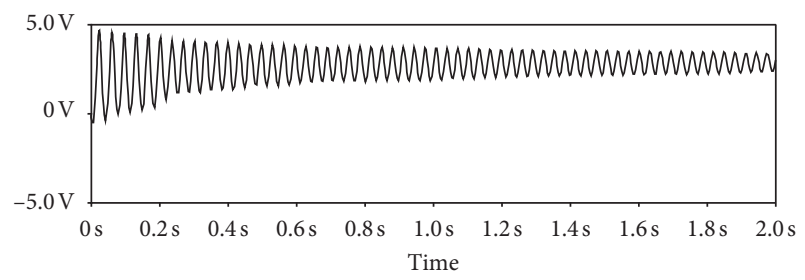

$\mathrm{V}(\mathrm{UC1}$ : OUT)

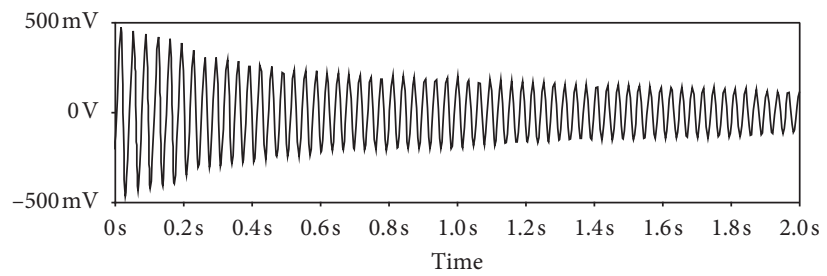

$\mathrm{V}(\mathrm{UC2}$ : OUT $)$

(a)

(b)

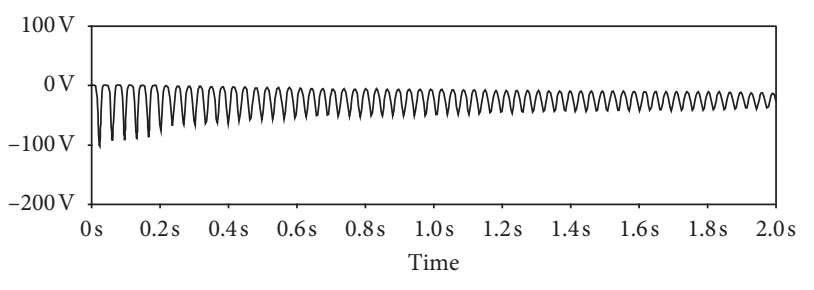

V(MULT2: OUT)

(c)

Figure 9: Output voltages of (a) amplifier with capacitor $C_{1}$, (b) amplifier with capacitor $C_{2}$, and (c) the nonlinear part $x^{3}$ for $R^{\prime} \leq 1 K \Omega$, $C_{1}=100 \mathrm{nF}, C_{2}=10 \mathrm{uF}, R=R_{1}=R_{2}=10 \mathrm{~K} \Omega, V_{c c}=12 \mathrm{~V}, R_{4}=5 \mathrm{~K} \Omega, R_{6}=2.77 \mathrm{~K} \Omega, R_{3}=R_{5}=5 \mathrm{~K} \Omega$.

and 9). In these environmental conditions, electrical oscillations are cared over time. But the signal's amplitude decreases continuously.

Other simulations have been carried out by varying resistors' values of $R_{4}$ and $R_{6}$. In general, the same behavior is obtained, So the behavior of the output voltages depends on the values of $R^{\prime}$. Results obtained from numerical investigations put forward spontaneous electrical activity consistent with self-sustained electrical oscillations during the dynamics of the microtubule's protofilament.

\section{Conclusion}

In this work, we tantalized to build an electrical analogue computer of MT's protofilament drawing from the partial differential equation of MT's motion. By using the suitable values of MT's parameters, we have been able to find suitable values of computing elements. Numerical results have shown that the behaviour of the output voltage generated by the electrical analogue computer is modulated by the resistance $R^{\prime}$. High values of $R^{\prime}$ promote increase in the behavior of the signal that can become linear for very high 
values of $R^{\prime}$ while low values of $R^{\prime}$ promote decrease in the behavior of the electrical voltage and care the oscillation over time. To build a more realistic analogue computer, one needs to use the exact values of MT parameters to derive the suitable computing elements. The behaviour of the output voltage obtained for $R^{\prime} \leq 1 \mathrm{~K} \Omega$ is similar to the results obtained by experimental studies. This study put forward the electrical activity of microtubule protofilament by electrical oscillations occurring at different regimes with amplitude changes as mechanism for intracellular signaling and communications inside neurons. So designing microtubule as an electrical analogue computer can be useful to better understand the dynamics of the entire system and to build new biological nanowires and biotransistors.

\section{Data Availability}

No data were used to support this study.

\section{Conflicts of Interest}

The authors declare that they have no conflicts of interest.

\section{References}

[1] J. Zimmerman, R. Parameswaran, and B. Tian, "Nanoscale semiconductor devices as new biomaterials," Biomaterial Science, vol. 2, no. 5, pp. 619-626, 2014.

[2] D. Sekulić and M. Satarić, "Microtubule as nanobioelectronic nonlinear circuit," Serbian Journal of Electrical Engineering, vol. 9, no. 1, pp. 107-119, 2012.

[3] C. Conde and A. Cáceres, "Microtubule assembly, organization and dynamics in axons and dendrites," Nature Reviews Neuroscience, vol. 10, no. 5, pp. 319-332, 2009.

[4] M. Stiess and F. Bradke, "Neuronal polarization: The cytoskeleton leads the way," Developmental Neurobiology, vol. 71, no. 6, pp. 430-444, 2011.

[5] A. Priel, A. J. Ramos, J. A. Tuszynski, and H. F. Cantiello, "The dendritic cytoskeleton as a computational device: An hypothesis," "The dendritic cytoskeleton as a computational device: An hypothesis," in The Emerging Physics of Consciousness, J. A. Tuszynski, Ed., Springer, Berlin, Germany, pp. 293-325, 2006.

[6] B. D. Johnson and L. Byerly, " $\mathrm{Ca} 2^{+}$channel $\mathrm{Ca}\left(2^{+}\right)$-dependent inactivation in a mammalian central neuron involves the cytoskeleton," Pflügers Archiv European Journal of Physiology, vol. 429, no. 1, pp. 14-21, 1994.

[7] D. Johnston, J. C. Magee, C. M. Colbert, and B. R. Christie, "Active properties of neuronal dendrites," "Active properties of neuronal dendrites," in The Structure and Function of Nervous Tissue, G. H. Bourne, Ed., pp. 205-267, Academic Press, Cambridge, MA, USA, 1996.

[8] A. Priel, A. J. Ramos, J. A. Tuszynski, and H. F. Cantiello, “A biopolymer transistor: Electrical amplification by microtubules," Biophysical Journal, vol. 90, no. 12, pp. 4639-4643, 2006.

[9] D. Havelka, M. Cifra, and O. Kučera, "Multi-mode electromechanical vibrations of a microtubule:In silicodemonstration of electric pulse moving along a microtubule," Applied Physics Letters, vol. 104, no. 24, pp. 1-4, Article ID 243702, 2014.
[10] J. Pokorný, J. Pokorný, and J. Kobilková, "Postulates on electromagnetic activity in biological systems and cancer," Integrative Biology, vol. 5, no. 12, pp. 1439-1446, 2013.

[11] S. R. Hameroff and R. Penrose, "Orchestrated reduction of quantum coherence in brain microtubules," "Orchestrated reduction of quantum coherence in brain microtubules," in Toward a Science of Consciousness, S. Hameroff, A. K. Kasszniak, and A. C. Scott, Eds., MIT Press, Cambridge, MA, USA, 1996.

[12] F. Daneshmand, E. Ghavanloo, and M. Amabili, "Wave propagation in protein microtubules modeled as orthotropic elastic shells including transverse shear deformations," Journal of Biomechanics, vol. 44, no. 10, pp. 1960-1966, 2011.

[13] F. Daneshmand, H. Farokhi, and M. Amabili, "A higher-order mathematical modeling for dynamic behavior of protein microtubule shell structures including shear deformation and small-scale effects," Mathematical Biosciences, vol. 252, pp. 67-82, 2014.

[14] F. Daneshmand and M. Amabili, "Coupled oscillations of a protein microtubule immersed in cytoplasm: An orthotropic elastic shell modeling," Journal of Biological Physics, vol. 38, no. 3, pp. 429-448, 2012.

[15] E. Ghavanloo, F. Daneshmand, and M. Amabili, "Vibration analysis of a single microtubule surrounded by cytoplasm," Physica E: Low-Dimensional Systems and Nanostructures, vol. 43, no. 1, pp. 192-198, 2010.

[16] E. Ghavanloo, F. Daneshmand, and M. Amabili, "Prediction of bending stiffness and deformed shape of non-axially compressed microtubule by a semi-analytical approach," Journal of Biological Physics, vol. 36, no. 4, pp. 427-435, 2010.

[17] S. Sahu, S. Ghosh, K. Hirata, D. Fujita, and A. Bandyopadhyay, "Multi-level memory-switching properties of a single brain microtubule," Applied Physics Letters, vol. 102, pp. 1-4, Article ID 123701, 2013.

[18] J. A. Tuszynski, T. J. A. Craddock, and E. J. Carpenter, "Bioferroelectricity at the nanoscale," Journal of Computational and Theoretical Nanoscience, vol. 5, no. 10, pp. 2022-2032, 2008.

[19] A. P. Kalra, S. D. Patel, A. F. Bhuiyan et al., "Investigation of the electrical properties of microtubule ensembles under celllike conditions," Nanomaterials, vol. 10, no. 2, p. 265, 2020.

[20] J. A. Tuszynski, D. Friesen, H. Freedman et al., "Microtubules as sub-cellular memristors," Scientific Reports, vol. 10, no. 1, pp. 1-11, 2020.

[21] B. C. Gutierrez, H. F. Cantiello, and M. del Rocío Cantero, "Electrical oscillations of brain microtubules," Biology, 2020.

[22] D. I. Ilić, M. V. Satarić, and N. Ralević, "Microtubule as a transmission line for ionic currents," Chinese Physics Letters, vol. 26, no. 7, Article ID 073101, 2009.

[23] S. Zdravković, L. Kavitha, M. V. Sataric, S. Zekovic, and J. Petrović, "Modified extended tanh-function method and nonlinear dynamics of microtubules," Chaos, Solitons \& Fractals, vol. 45, no. 11, pp. 1378-1386, 2012.

[24] S. Zdravkovic, M. V. Sataric, A. Maluckov, and A. Balaz, "A nonlinear model of the dynamics of radial dislocations in microtubules," Applied Mathematics and Computation, vol. 237, no. 15, pp. 227-237, 2014. 